\title{
Sterile Leukocytosis Predicts Hemorrhagic Transformation in Arterial Ischemic Stroke: A National Inpatient Sample Study
}

\author{
Aldana M. Antoniazzi ${ }^{1}$, Santiago R. Unda ${ }^{2}$, Daniel M. Klyde ${ }^{1}$, Raphael Miller ${ }^{1}$, Sharon Lam ${ }^{1}$, Rose Fluss \\ 1 , David J. Altschul ${ }^{3}$ \\ 1. Neurological Surgery, Montefiore Medical Center, New York, USA 2. Neurological Surgery, Montefiore Medical \\ Center, Bronx, USA 3. Neurological Surgery, Albert Einstein College of Medicine, Bronx, USA
}

Corresponding author: Santiago R. Unda, santiagounda94@gmail.com

\begin{abstract}
Objective: Hemorrhage transformation (HT) is a known complication of arterial ischemic stroke (AIS). In addition, it is known that the increase of proinflammatory immune cells in the brain tissue after AIS predict worse outcomes. However, it is not clear whether inflammation due to preceding or post-stroke infections affect outcomes and moreover, if systemic inflammatory markers could be useful as a clinical prediction tool for HT post-stroke. Therefore, our objective was to assess the association between systemic proinflammatory profile in AIS patients with HT and in-hospital mortality that did not course with acute infections during hospitalization.
\end{abstract}

Methods: This study was conducted using the 2016 and 2017 National Inpatient Sample (NIS) with International Classification of Diseases (ICD-10) codes. Multivariate logistic regression was used to examine the association between HT and in-hospital mortality with pro-inflammatory anomalies of white blood cells (WBCs) in AIS patients. Exclusion criteria comprised patients with under 18 years old, and with a diagnosis of gastrointestinal, urogenital, respiratory infection, bacteremia, viral infection, sepsis, or fever.

Results: A total of 212,356 patients with AIS were included in the analysis. 422 (0.2\%) patients had a HT and 10,230 (4.8\%) patients died during hospitalization. The most common WBC pro-inflammatory marker was leukocytosis with $6.9 \%(n=29 / 422)$ of HT and $5.5 \%(n=560 / 10,230)$ of patients that died during hospitalization. After adjusting for socio-demographic, comorbidities and treatment factors, leukocytosis was found to be an independent risk factor for both outcomes, HT [OR = 1.5, 95\% CI: 1-2.3, p=0.024] and, inhospital mortality [OR = 1.5, 95\% CI: $1.3-1.6, \mathrm{p}<0.001]$.

Conclusion: Sterile leukocytosis is a potential clinical prediction tool to determine which patients are at higher risk of developing HT and die during hospitalization.

Review began 05/04/2021 Review ended 05/06/2021 Published 05/11/2021

๑) Copyright 2021

Antoniazzi et al. This is an open access article distributed under the terms of the Creative Commons Attribution License CC-BY 4.0., which permits unrestricted use, distribution, and reproduction in any medium, provided the original author and source are credited.
Categories: Neurology, Neurosurgery

Keywords: leukocytosis, systemic inflammation, hemorrhagic transformation, stroke, nationwide inpatient sample (nis)

\section{Introduction}

Arterial ischemic stroke (AIS) is the fifth leading cause of death in the United States [1]. Although thrombolysis is a standard treatment commonly used in acute AIS patients [2], this procedure has known complications - the major one being hemorrhagic transformation (HT), which is strongly associated with increased morbidity and mortality [3,4]. Among the pathophysiological mechanisms that promote the dynamic and complex HT phenomenon, the blood-brain barrier (BBB) disruption and brain damage driven by systemic inflammation has shown a prominent role [5].

After AIS onset, the activation of the immune cells such as leukocytes, microglia, mast cells and astrocytes release substances including cytokines, proteases and, reactive oxygen species (ROS) making the BBB more permeable and at the same time, blocking cerebral microvasculature blood flow [6-8]. Through the disrupted $\mathrm{BBB}$, peripheral immune cells enter the brain parenchyma and enhance the ongoing neuroinflammatory process. Thus, in the postischemic inflammatory sequence of events involving the brain, its vessels, circulating blood, and lymphoid organs, systemic inflammation contributes to initiating but also to aggravating the stroke response [9-11].

Despite the increase in white blood cells (WBCs) count in AIS patients has been largely associated with mortality [12], the clinical relevance of systemic inflammatory biomarkers in HT has been addressed without considering the influence of infections, mostly in single-center studies with limited sample sizes and patients' heterogeneity; thus, its predictive value remains largely unknown. Therefore, in this study we sought to explore the relationship of these variables using a large-scale database, the National Inpatient 


\section{Materials And Methods}

\section{Data source}

This retrospective cohort study used the NIS database. This large database contains a $20 \%$ stratified sample of all hospital discharges in states participating in the Healthcare Cost and Utilization Project (HCUP) in the U.S. It contains demographic, diagnostic, and procedural data, with diagnoses and procedures captured in the form of the International Classification of Diseases 10th edition (ICD-10).

\section{Study sample}

Primary inclusion criteria were patients with Stroke (ICD-10th: I66) from January 1, 2016 to December 31, 2017. Exclusion criteria comprised: 1) patients under 18 years old and/or 2) patients with a diagnosis of gastrointestinal, urogenital, respiratory infection, bacteremia, viral infection, sepsis, or fever.

\section{Collected data}

Examined data comprised demographics including age, sex, race, and median household income categorized in quartiles. Comorbidities analyzed included hypertension, diabetes mellitus with and without complications, congestive heart failure, renal disease, myocardial infarction, chronic pulmonary disease, peripheral vascular disease, status post-administration of recombinant tissue plasminogen activator (rtPA), thrombectomy, National Institute of Health Stroke Scale (NIHSS) Score and history of cerebrovascular disease (see Appendix).

Finally, we selected the most prevalent pro-inflammatory WBC anomalies found in the NIS 2016 and 2017 database, this included eosinophilia, lymphocytosis, monocytosis, plasmacytosis, bandemia, and elevated WBC count (leukocytosis). All codes for this study are shown in the supplementary material (see Appendix).

\section{Outcomes}

The primary outcome analyzed for patients with AIS was the development of hemorrhage transformation (HT) (ICD-10th: I61-I62.9). Our secondary outcome analyzed was death during hospitalization (in-hospital mortality).

\section{Statistical analysis}

Interactions between specific types of pro-inflammatory WBC anomalies and outcomes were assessed. A univariate analysis was done to identify unevenly distributed variables in the WBC anomalies that could be playing as confounding factors; for this step, all continuous values were represented using mean \pm standard deviation (SD) or median and interquartile range (IQR). Categorical variables were described using frequencies and proportions. Comparisons were performed using Student's t-test, the nonparametric MannWhitney test or $\chi^{2}$ tests as appropriate.

Factors with a p-value less than 0.10 were then included in a multiple logistic regression analysis to reflect the adjusted association between WBC anomalies and each of the primary outcomes. Odds ratios (OR) and $95 \%$ confidence intervals (CI) were calculated. A p-value less than 0.05 was considered statistically significant. SPSS v.24 software (IBM, Armonk, NY) was used to perform the statistical analysis.

\section{Results}

A total of 260,189 patients with AIS were captured in the 2016 \& 2017 NIS database. Out of these patients, 47,833 (18.4\%) were excluded due to a diagnosis related to fever or infections. Therefore, 212,356 patients were included for the main analysis. We identified $422(0.2 \%)$ patients with HT, and 10,230 (4.8\%) that died during hospitalization. At least one of the proinflammatory WBC parameters were found in $7.3 \%(n=31)$ of the HT patients and in $5.6 \%(n=571)$ of patients that died during hospitalization, the most prevalent anomaly was leukocytosis with $6.9 \%(n=29)$ and $5.5 \%(n=560)$ in HT and died during hospitalization, respectively (Figure 1). 


\section{Cureus}

Proinflammatory

WBC anomalies in AIS patients

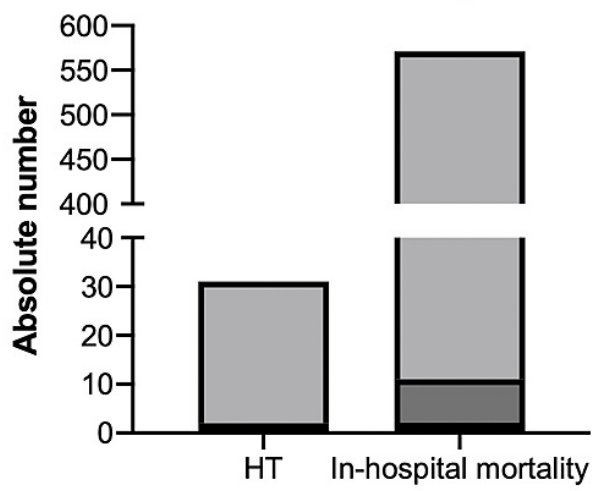

FIGURE 1: Proinflammatory white blood cell (WBC) anomalies in AIS patients.

Total number of AIS patients found in the NIS 2016 and 2017 with WBC anomalies and with hemorrhagic transformation (HT) and/or died during hospitalization.

AIS - Arterial ischemic stroke

$0.2 \%$ of patients had eosinophilia and $0.2 \%$ had bandemia in the HT cohort, while in patients that died inhospital $0.02 \%$ had eosinophilia and $0.09 \%$ bandemia. The rest of WBC anomalies represented the $0 \%$ of the cases.

We proceed with a univariate analysis to assess unevenly distributed variables in patients with normal WBC and leukocytosis (Table 1).

\section{Variables}

Age in years at admission, mean (SD)

Female, n (\%)

Race, n (\%)

White

African-American

Hispanic

Others

Household income, n (\%)

Quartile 1

Quartile 2

Quartile 3

Quartile 4

Hypertension, n (\%)

Diabetes simple, n (\%)

Diabetes complicated, $\mathrm{n}(\%)$

Congestive heart failure, n (\%)
White Blood Cells Count

Leukocytosis $(n=7,307)$

Normal $(n=205,046)$

P-value

67 (15)

69 (15)

$<0.001^{*}$

3,488 (47.7)

97,674 (47.6)

0.868

5,049 (71.8)

135,801 (68.7)

927 (13.2)

34,199 (17.3)

15,797 (8)

$11,734(6)$

$457(6.5)$

$2,206(30.7)$

62,494 (31)

1,904 (26.5)

52,531 (26.1)

$48,036(23.6)$

0.787

1,723 (24)

38,543 (19.1)

152,305 (74.3)

0.537

61,994 (30.2)

0.586

$2,231(30.5)$

$779(10.7)$

$24,981(12.2)$

$<0.001^{*}$

1,373 (18.8)

38,720 (18.9)

0.841 


\section{Cureus}

\begin{tabular}{|c|c|c|c|}
\hline Renal disease, $n$ (\%) & $1,282(17.5)$ & $38,720(18.1)$ & 0.262 \\
\hline Myocardial infarction, n (\%) & $949(13.0)$ & 22,282 (10.9) & $<0.001^{*}$ \\
\hline Chronic pulmonary disease, $\mathrm{n}(\%)$ & $1,286(17.6)$ & 33,949 (16.6) & $0.019^{*}$ \\
\hline Peripheral vascular disease, $\mathrm{n}(\%)$ & 846 (11.6) & $21,556(10.5)$ & $0.004^{*}$ \\
\hline Status post administration of tPA, $n(\%)$ & $283(3.9)$ & $5,903(2.9)$ & $<0.001^{*}$ \\
\hline Thrombectomy, n (\%) & $458(6.3)$ & $8,478(4.1)$ & $<0.001^{*}$ \\
\hline \multicolumn{4}{|l|}{ NHISS score, n (\%) } \\
\hline $0-9$ & $664(9.1)$ & $2,3710(11.6)$ & $<0.001^{*}$ \\
\hline 10-19 & $284(3.9)$ & $5,064(2.5)$ & $<0.001^{*}$ \\
\hline $20-29$ & $159(2.2)$ & 2,203 (1.1) & $<0.001^{*}$ \\
\hline 30-39 & $31(0.4)$ & $306(0.1)$ & $<0.001^{*}$ \\
\hline $40-42$ & $1(0.0)$ & $19(0.0)$ & 0.702 \\
\hline \multicolumn{4}{|l|}{ Localization, n (\%) } \\
\hline \multicolumn{4}{|l|}{ Extracranial } \\
\hline Unspecified & $59(0.8)$ & $1,611(0.8)$ & 0.836 \\
\hline Vertebral artery & $140(1.9)$ & 2,878 (1.4) & $<0.001^{*}$ \\
\hline Basilar artery & $89(1.2)$ & $2,125(1.0)$ & 0.133 \\
\hline Carotid artery & $467(6.4)$ & $10,918(5.3)$ & $<0.001^{*}$ \\
\hline \multicolumn{4}{|l|}{ Intracranial } \\
\hline Unspecified & 409 (5.6) & $11,531(5.6)$ & 0.924 \\
\hline Middle cerebral artery & $1,856(25.4)$ & 38,824 (18.9) & $<0.001^{*}$ \\
\hline Anterior cerebral artery & $155(2.1)$ & $3,001(1.5)$ & $<0.001^{*}$ \\
\hline Posterior cerebral artery & $1,856(25.4)$ & $38,824(18.9)$ & $<0.001^{*}$ \\
\hline Cerebellar & $259(3.5)$ & $5,286(2.6)$ & $<0.001^{*}$ \\
\hline
\end{tabular}

TABLE 1: Univariate analysis of patients with/without leukocytosis in the NIS database, 2016 and 2017.

*Included in multivariate analysis

NIS - National inpatient sample

Variables with $\mathrm{p}<0.1$ included age, race, complicated diabetes mellitus, myocardial infarction, chronic pulmonary disease, peripheral vascular disease, thrombolysis with rtPA, patients with NIHSS score, and vertebral artery, carotid artery, middle cerebral artery, anterior cerebral artery, posterior cervical artery, and cerebellar localizations. All mentioned variables were included to adjust the multiple logistic regression models.

After adjusting for variables with $\mathrm{p}<0.1$ in the univariate analysis, the multiple logistic regression model revealed that leukocytosis is an independent risk factor for developing HT [OR $=1.5,95 \%$ CI: 1-2.3, $p=0.024]$ as well as increased the risk of in-hospital mortality [OR $=1.5,95 \%$ CI: $1.3-1.6, \mathrm{p}<0.001]$ in AIS patients (Figures $2 A, 2 B$ ). 

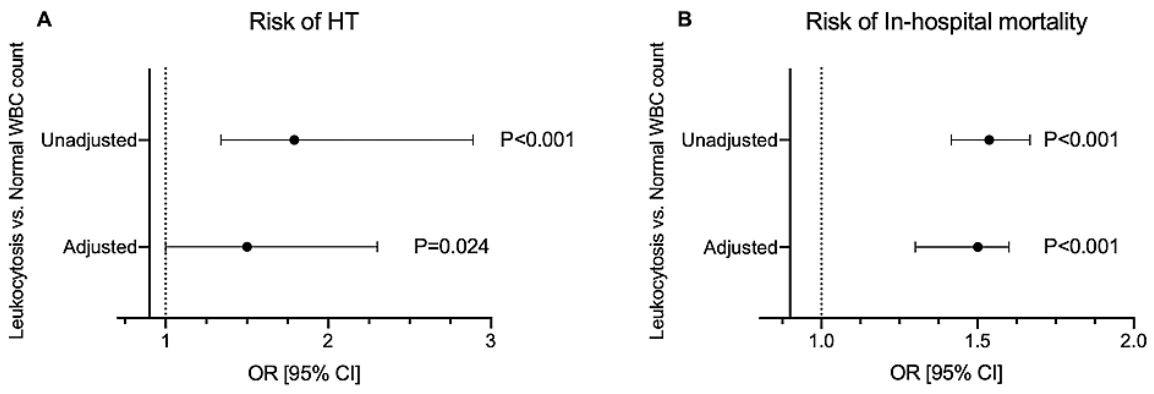

FIGURE 2: Risk of hemorrhagic transformation (HT) and in-hospital mortality in patients with leukocytosis.

(A) Crude and adjusted odds ratio (OR) with $95 \%$ confidence interval $(95 \% \mathrm{Cl})$ of leukocytosis with risk of $\mathrm{HT}$ following AIS. (B) Crude and adjusted OR with $95 \% \mathrm{Cl}$ of leukocytosis with risk of in-hospital mortality in AIS patients.

AIS - Arterial ischemic stroke

\section{Discussion}

In our study, we analyzed the association of systemic pro-inflammatory WBC markers and HT in patients with AIS. We found that leukocytosis increases 1.5 -fold times the risk of HT independently of sociodemographic, comorbidities, and treatment factors. Furthermore, our results support previous evidence regarding the higher risk of in-hospital mortality in AIS with leukocytosis. In addition, our analyses excluded patients coursing urinary tract infections, lung infections, gastrointestinal infections, sepsis, and/or fever. Therefore, we report an adjusted large national-scale study with important implications for physicians taking care of AIS patients that are not coursing an active infection or secondary cause of systemic inflammation.

Despite HT is a serious complication commonly following reperfusion therapy and, sometimes as a result of the natural evolution of the disease [13], the current treatment recommendations include anticoagulants, thrombolysis, and intravascular procedures [14]. Thus, available and routine clinical prediction tools of HT based on pathophysiological mechanisms are necessary to decide the best management options according to the patient's system response to stroke. In this context, experimental studies have clarified that WBC has a critical role in post-ischemic neuroinflammation and secondary neurovascular injury [15]. Within 4 to $6 \mathrm{~h}$ after AIS onset, circulating leukocytes adhere to the vessel wall, transmigrate through the BBB and enter the brain, subsequently releasing pro-inflammatory mediators, resulting in a secondary lesion in the penumbra surrounding the nucleus of the ischemia [8]. Neutrophils are the earliest WBC subtype to infiltrate the ischemic brain [16]. These immune cells exacerbate the injury by releasing additional inflammatory factors, such as ROS, MMP-9, and neutrophil elastase, which are prominent effectors of BBB disruption [17]. It also has been shown that most AIS patients course some degree of immune dysregulation; however, when these mechanisms are overstimulated worsen short and long-term clinical outcomes are expected [18-20].

Regarding the clinical evidence in this matter, in spite most of the reports that have shown an association of leukocytes subtypes with increased risk of HT have not taken into account infections, a recent study by Semerano et al. [21] showed that increasing leukocytes predicts worsen outcomes post-AIS, including HT independently of preceding or early post-stroke infections, suggesting that stroke-evoked sterile inflammation is a major key to understand patients' hospital course and prognosis. Even though in the mentioned study the authors treated the WBC as continuous variables, their findings showed that high leukocyte counts increase 1.4 times the odds of HT, which is in line with our findings. In addition, our results showed an increased risk of in-hospital mortality in patients with leukocytosis which supports previous studies $[12,22]$ and hence, strongly suggests that AIS patients with evidence of sterile inflammation may benefit from complementary management therapies such as closer monitoring and anti-inflammatories from early stages.

Although we consider our results are encouraging since it is the first demonstration, to our best knowledge, of the potential suggestive value of a routine laboratory marker for a serious complication in AIS using a large-national database, limitations inherent to the retrospective nature of our work need to be mentioned. Our variables of interest, WBC, are presented in the NIS database as categorical variables instead of continuous values and therefore, it was not possible to identify a threshold that could increase the sensitivity and specificity to predict HT and mortality. Moreover, this study does not elucidate the essential 


\section{Cureus}

causes and mechanisms by which systemic inflammation put in a higher risk of worsening outcomes. Thus, further clarification of mechanisms, cut-off values, and specific leukocyte subtypes is needed.

\section{Conclusions}

Peripheral leukocytosis is an independent predictor of HT and in-hospital mortality in AIS patients that did not course with a pre/post-AIS infection or other sources of systemic inflammation besides the attributed AIS-induced inflammation. Hence, this national study supports previous experimental and clinical evidence regarding the role of sterile inflammation in stroke pathophysiology and as a potential predictive biomarker. Nevertheless, further validation regarding the dynamic change of WBC during the acute and subacute postAIS phases and the role of other proinflammatory molecules are needed to incorporate the concept of sterile leukocytosis in clinical reasoning.

\section{Appendices}

Variables included

Arterial ischemic stroke

Hemorrhagic transformation

Eosinophilia

Lymphocytosis (symptomatic)

Monocytosis (symptomatic)

Plasmacytosis

Bandemia

Elevated white blood cell count

Hypertension

Diabetes mellitus simple

Diabetes mellitus complicated

Lipidemias

Obesity

Congestive heart failure

Cerebrovascular disease

Depression

Current smoker

Sleep Apnea

Status post-administration of recombinant tissue plasminogen activator (rtPA)

Thrombectomy

National Institute of Health Stroke Scale (NIHSS)
ICD-10

166

$161-162.9$

D72.1

D72.820

D72.821

D72.822

D72.825

D72.828, D72.829

110

E10, E11, E12, E13, E14

E10, E11, E12, E13, E14

E78

E66, Z683, Z684,

I099, I110, I13, I255, I42, I43, I50, P290

G45, G46, H340, 16

F32, F33

F17

G47.3

Z92.82

037J3, 037G3

R29.7

TABLE 2: ICD-10 codes used for the analysis of peripheral white blood cell abnormalities in AIS patients discharged in the NIS 2016 and 2017 dataset.

ICD-10 - International Classification of Diseases

AIS - arterial ischemic stroke

NIS - National Inpatient Sample 


\section{Disclosures}

Human subjects: All authors have confirmed that this study did not involve human participants or tissue. Animal subjects: All authors have confirmed that this study did not involve animal subjects or tissue. Conflicts of interest: In compliance with the ICMJE uniform disclosure form, all authors declare the following: Payment/services info: All authors have declared that no financial support was received from any organization for the submitted work. Financial relationships: All authors have declared that they have no financial relationships at present or within the previous three years with any organizations that might have an interest in the submitted work. Other relationships: All authors have declared that there are no other relationships or activities that could appear to have influenced the submitted work.

\section{References}

1. Mozaffarian D, Benjamin EJ, Go AS, et al.: Heart disease and stroke statistics-2016 update: a report from the American Heart Association. Circulation. 2016, 133:e38-60. 10.1161/CIR.0000000000000350

2. Robinson T, Zaheer Z, Mistri AK: Thrombolysis in acute ischaemic stroke: an update . Ther Adv Chronic Dis. 2011, 2:119-31. 10.1177/2040622310394032

3. Boisseau W, Fahed R, Lapergue B, et al.: Predictors of parenchymal hematoma after mechanical thrombectomy: a multicenter sudy. Stroke. 2019, 50:2364-70. 10.1161/STROKEAHA.118.024512

4. Jickling GC, Liu D, Stamova B, Ander BP, Zhan X, Lu A, Sharp FR: Hemorrhagic transformation after ischemic stroke in animals and humans. J Cereb Blood Flow Metab. 2014, 34:185-99. 10.1038/jcbfm.2013.203

5. Marta-Enguita J, Navarro-Oviedo M, Rubio-Baines I, et al.: Association of calprotectin with other inflammatory parameters in the prediction of mortality for ischemic stroke. J Neuroinflammation. 2021, 18:3. 10.1186/s12974-020-02047-1

6. Shi K, Tian DC, Li ZG, et al.: Global brain inflammation in stroke. Lancet Neurol. 2019, 18:1058-66. 10.1016/S1474-4422(19)30078-X

7. Anrather J, Iadecola C: Inflammation and stroke: an overview. Neurotherapeutics. 2016, 13:661-70. 10.1007/s13311-016-0483-X

8. Ahmad M, Graham SH: Inflammation after stroke: mechanisms and therapeutic approaches. Transl Stroke Res. 2010, 1:74-8. 10.1007/s12975-010-0023-7

9. Xu X, Jiang Y: The yin and Yang of innate immunity in stroke . Biomed Res Int. 2014, 2014:807978. 10.1155/2014/807978

10. Iadecola C, Anrather J: The immunology of stroke: from mechanisms to translation . Nat Med. 2011, 17:796808. 10.1038/nm.2399

11. Lindsberg PJ, Strbian D, Karjalainen-Lindsberg ML: Mast cells as early responders in the regulation of acute blood-brain barrier changes after cerebral ischemia and hemorrhage. J Cereb Blood Flow Metab. 2010, 30:689-702. 10.1038/jcbfm.2009.282

12. Abburi N, Sterenstein A, Breit H, Song S, John S, Da Silva I, Koffman L: Description of an association between leukocytosis and clinical outcomes in large hemispheric infarctions. J Stroke Cerebrovasc Dis. 2021, 30:105614. 10.1016/j.jstrokecerebrovasdis.2021.105614

13. Pande SD, Win MM, Khine AA, Zaw EM, Manoharraj N, Lolong L, Tin AS: Haemorrhagic transformation following ischaemic stroke: a retrospective study. Sci Rep. 2020, 10:5319. 10.1038/s41598-020-62230-5

14. Mullhi RK, Singh N, Veenith T: Critical care management of the patient with an acute ischaemic stroke . Br J Hosp Med (Lond). 2021, 82:1-9. 10.12968/hmed.2020.0123

15. Joya A, Ardaya M, Montilla A, et al.: In vivo multimodal imaging of adenosine A1 receptors in neuroinflammation after experimental stroke. Theranostics. 2021, 11:410-25. 10.7150/thno.51046

16. Rolfes L, Riek-Burchardt M, Pawlitzki M, et al.: Neutrophil granulocytes promote flow stagnation due to dynamic capillary stalls following experimental stroke. Brain Behav Immun. 2021, 93:322-30. 10.1016/j.bbi.2021.01.011

17. Yang C, Hawkins KE, Doré S, Candelario-Jalil E: Neuroinflammatory mechanisms of blood-brain barrier damage in ischemic stroke. Am J Physiol Cell Physiol. 2019, 316:C135-53. 10.1152/ajpcell.00136.2018

18. Montellano FA, Ungethüm K, Ramiro L, et al.: Role of blood-based biomarkers in ischemic stroke prognosis: a systematic review. Stroke. 2021, 52:543-51. 10.1161/STROKEAHA.120.029232

19. Tian M, Li Y, Wang X, et al.: The hemoglobin, albumin, lymphocyte, and platelet (HALP) score Is associated with poor outcome of acute ischemic stroke. Front Neurol. 2020, 11:610318. 10.3389/fneur.2020.610318

20. Hu D, Ding C, Jiang X, et al.: Elevated levels of inflammation markers predict poor outcomes in acute ischemic stroke patients after intravenous thrombolysis. J Stroke Cerebrovasc Dis. 2021, 30:105587. 10.1016/j.jstrokecerebrovasdis.2020.105587

21. Semerano A, Strambo D, Martino G, Comi G, Filippi M, Roveri L, Bacigaluppi M: Leukocyte counts and ratios are predictive of stroke outcome and hemorrhagic complications independently of infections. Front Neurol. 2020, 11:201. 10.3389/fneur.2020.00201

22. Chen J, Zhang FL, Lv S, et al.: Association of admission leukocyte count with clinical outcomes in acute ischemic stroke patients undergoing intravenous thrombolysis with recombinant tissue plasminogen activator. Curr Neurovasc Res. 2020, 17:660-6. 10.2174/1567202617999201125201616 